\title{
Better Mood, Better Outcomes in Stroke Elderly Patients?
}

ISSN: 2578-0093

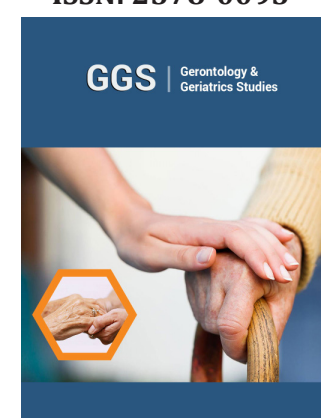

*Corresponding author: Gilberto A Jiménez Castillo, Neurological Geriatrics, Mexico

Submission: 㘹January 12, 2021

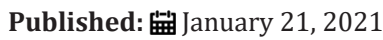

Volume 6 - Issue 4

How to cite this article: Gilberto A Jiménez Castillo, Antonio Anaya Escamilla, Luis E Martínez Bravo, Arturo Márquez Ortiz. Better Mood, Better Outcomes in Stroke Elderly Patients?. Gerontol \& Geriatric stud. 6(4). GGS. 000643. 2021. DOI: 10.31031/GGS.2021.06.000643

Copyright@ : Gilberto A Jiménez Castillo, This article is distributed under the terms of the Creative Commons Attribution 4.0 International License, which permits unrestricted use and redistribution provided that the original author and source are credited.

\author{
Gilberto A Jiménez Castillo ${ }^{1,2,3 *}$, Antonio Anaya Escamilla1, Luis E Martínez \\ Bravo $^{4}$ and Arturo Márquez Ortiz ${ }^{2}$
}

${ }^{1}$ Neurological Geriatrics, Mexico

${ }^{2}$ Tecnologico de Monterrey, Mexico

${ }^{3}$ Hospital General y Medicina Familiar de Zona No. 2, Instituto Mexicano del Seguro Social, Monterrey, Mexico

${ }^{4}$ Hospital General de Zona No. 4, Instituto Mexicano del Seguro Social, Guadalupe, Mexico

\section{Opinion}

Stroke is one of the main global causes of death and disability-adjusted life-years around the world [1]. Most of community-dwelling elderly stroke survivors need special care on their daily living [2]. Stroke treatment in elderly patients is complex. Many randomized clinical trials exclude these individuals for their multiple geriatric syndromes, functional status and comorbidities [3]. Nowadays, prevention is the principal intervention, in particular lifestyle changes and control of chronic diseases. Ischemic stroke treatment is focused on early management; recognition of eligible patients for intravenous thrombolysis or mechanical thrombectomy, early rehabilitation and initiation secondary stroke prevention. Classically, antiplatelet and anticoagulants are prescribed for secondary stroke prevention due to their inhibition of thrombus formation [4]. Although, rehabilitation is a focal treatment for disability and motor recovery, there is major interest in supportive novel treatment pathways. One that has been booming recently is the serotonin signaling system and the selective serotonin reuptake inhibitors (SSRIs) administration after an acute ischemic stroke.

Descriptions of cellular pathways for neurogenesis and clinical recovery manifestations have been studied. At a cellular level, neurogenesis is a rare term attributed or used for drugs now a day. Thus, theories have recognized and reviewed SSRIs efficacy for these traits. Neurotrophic growth factors have been implicated. Their expression in peri-infarct matter leads to migration and maturation of neurons from the sub granular zone in the hippocampus. Brain derived neurotropic factor induction enhances neuroplasticity. In addition, SSRIs incite a reinstatement of neuronal organization by inhibition of overexcited neuronal circuits caused by stroke. Moreover, inhibition of serotonin reuptake and higher levels of neurotransmitter in the synaptic cleft, modulate glutamate, calcium and $\gamma$-aminobutyric acid transmission for long-term potential restoration [5,6].

SSRIs are extensively used around the world and very common in the elder population. Mostly, small clinical trials suggest encouraging outcomes in different domains such a motor skills, depression and mortality. Consistency has not permitted SSRIs to be included as a routinely practice for ischemic stroke. For the moment, there are no clear indications to prescribe them regularly. Additionally, studies that focus on aging patients are scarce or nonexistent. Should there be broadened awareness for SSRIs during evaluation of elderly stroke patients?

Post-stroke depression appears in $30 \%$ of patients, and as we know, it can bring detrimental outcomes on quality of life, independence, and mobility. Meta-analysis shows consistent results for prevention. In 2020, Zhou et al. [7] included 3768 patients without depression from 4 trials, the analysis revealed positive outcomes in prevention of post- 
stroke depression (RR 0.78 [95\% CI 0.67-0.90; p .001) and low heterogeneity ( $12=23 \%$ ) [7]. Previous systemic reviews have also yield encouraging results [8].

Clinically, the most debated effect for SSRIs is motor recovery. One of the first double blinded placebo-controlled studies to demonstrate effectiveness was the FLAME study (Fluoxetine for motor recovery after acute ischemic Stroke), where the antidepressant group had a significant improvement in comparison to the placebo group in the Fugl-Meter motor scale (mean 34.0 points [95\% CI 29.7-38.4] vs 24.3 points [19.9-28.7]; p .003) [9]. Systemic reviews have assessed the use of SSRIs. The overall results do not identified benefits. When high bias studies are included, improvement is demonstrated. However, as previously mentioned, age is not often accounted for. We could only find that a systemic review by Mead et al. [10] mentioned that mostly recruited patients were in the range of $60-70$ years. Although, a high risk of bias is described, evidence of favorable outcomes is shown [10]. Interestingly, a study showed reduction in 30-day all-cause mortality with the administration of early treatment (first 5 days after the event) [11].

Evidence in the elderly to support the administration of SSRIs is scarce as previously mentioned. Could the described potential benefits of SSRIs out weight the side effects? For its low cost, should antidepressant treatment be initialized in selected candidates? Should the suspension occur only in the event of an adverse reaction? As SSRIs have shown to be safe, small doses could be initiate and titrated until clinical benefit. The choice must be individualized based in patient's profile. However, in a geriatric perspective, the fewer the anti-cholinergic effects the better. Anticholinergic activity leads easier adverse reactions (dry mouth, urinary retention, constipation, etc.) leads to worse outcomes. Uncertainty may rise on the duration of treatment, based on poststroke depression analysis, at least for 8 weeks could be beneficial.

At last, utilization of SSRIs on specific demographic areas or third world countries such as ours, may bring an additional tool for patient recovery. Further trials will define improvements and longterm answers for the elderly population, but for now, as the lines of investigation are open, consideration for SSRIs may be exciting.

\section{Conflict of Interest}

Authors have nothing to disclose with regard to commercial support.

\section{References}

1. Gorelick PB (2019) The global burden of stroke: persistent and disabling. Lancet Neurol 18(5): 417-418.

2. Skolarus LE, Freedman VA, Feng C, Wing JJ, Burke JF (2016) Care received by elderly us stroke survivors may be underestimated. Stroke 47(8): 2090-2095.

3. Heitsch LE, Panagos PD (2013) Treating the elderly stroke patient: complications, controversies and best care metrics. Clin Geriatr Med 29(1): 231-255.

4. Powers WJ, Rabinstein AA, Ackerson T, Adeoye OM, Bambakidis NC, et al. (2019) Guidelines for the early management of patients with acute ischemic stroke: 2019 update to the 2018 guidelines for the early management of acute ischemic stroke: a guideline for healthcare professionals from the American Heart Association/American Stroke Association. Stroke 50: e344-e418.

5. Elzib H, Pawloski J, Ding Y, Asmaro K (2019) Antidepressant pharmacotherapy and poststroke motor rehabilitation: A review of neurophysiologic mechanisms and clinical relevance. Brain Circ 5(2): 62-67.

6. Pinto CB, Velez FGS, Lopes F, Piza PVT, Dipietro L, et al. (2017) SSRI and motor recovery in stroke: reestablishment of inhibitory neural network tonus. Front Neurosci 11: 637.

7. Zhou S, Liu S, Liu X, Zhuang W (2020) Selective serotonin reuptake inhibitors for functional independence and depression prevention in early stage of post-stroke: A meta-analysis. Medicine (Baltimore) 99(6): e19062.

8. Legg LA, Tilney R, Hsieh CF, Wu S, Lundström E, et al. (2019) Selective serotonin reuptake inhibitors (SSRIs) for stroke recovery. Cochrane Database Syst Rev 26(11): CD009286.

9. Chollet F, Tardy J, Albucher JF, Thalamas C, Berard E, et al. (2011) Fluoxetine for motor recovery after acute ischaemic stroke (FLAME): a randomised placebo-controlled trial. Lancet Neurol 10(2): 123-130.

10. Mead GE, Hsieh CF, Lee R, Kutlubaev M, Claxton A, et al. (2013) Selective serotonin reuptake inhibitors for stroke recovery: a systematic review and meta-analysis. Stroke 44(3): 844-850.

11. Mortensen JK, Johnsen SP, Larsson H, Andersen G (2015) Early antidepressant treatment and all-cause 30-day mortality in patients with ischemic stroke. Cerebrovasc Dis 40(1-2): 81-90. 